EPiC Series in Language and Linguistics
Volume 2, 2017, Pages 295-303
Professional and Academic Discourse:
an Interdisciplinary Perspective

\title{
A Corpus-based Analysis of Non-Standard English Features in the Microblogging Platform Tumblr
}

\author{
Rosana Villares \\ Universidad de Zaragoza, Spain. \\ rosanavillares92@gmail.com
}

\begin{abstract}
Linguistic deviations from the standard use of English in computer-mediated communication have been described by the literature as distinctive Netspeak features. Using corpus linguistics and register theory, I examine those linguistic deviations in a corpus extracted from the microblogging platform Tumblr to describe how language use is shaped by the Internet medium. The sample of grammatical, discourse and style features analysed included the use of personal pronouns, idiomatic expressions, abbreviations, examples, quotations, repetitions, intensifiers, emotional and offensive language, as well as other features such as break and run-on-sentences, typography, punctuation, and multimodal elements. The corpus analysis reveals that these texts are characterised as being mainly short written messages combining typical features of the written mode of communication and features of extemporaneous, spoken discourse. Findings also show a non-standard use of punctuation and typography, the inclusion of multimedia elements that helps to overcome the lack of immediate feedback and the use of non-segmental phonology in the conversation. The results of the analysis suggest that Netspeak (non-standard) linguistic features are determined by context and are thus more likely to appear in digital spheres such as social networks, blogs and chats, among users who engage in one-to-one conversations or users who belong to the same in-group, and in communicative situations in which hobbies and personal experiences are the most frequently discussed topics.
\end{abstract}

\section{Internet Language and Netspeak Register}

During the last decades, the fast advancement of the Internet has offered platforms for new genres and styles of communication that have led to a linguistic freedom never known before outside the digital medium (ElBekaroui, 2008; Kleinman, 2010; Tagliamonte, 2008). Research regarding the linguistic features of Computer-mediated communication (CMC) refers to it as written speech because 
"Netspeak", as coined by David Crystal (2001), contains features of both modes of communication. Speech is characterised by a heavy use of first and second person pronouns, contractions, its level of formality is generally low and it can be rude. In writing, interlocutors are physically separated, feedback is asynchronous, the medium is durable, and participants commonly use a wide range of lexical choices and complex syntax (Baron, 2010). Furthermore, Netspeak introduces new linguistic features only visible in the digital medium. Unlike Crystal, who regards them as an expansion of the expressive richness of language, such creative and innovative ways have been criticised as deviant spelling even if users actually expect to receive some misspelled messages because of the speed and simplification factors the system brings with it (ElBekraoui, 2008). In addition to technical aspects, the introduction of linguistic variations and written strategies to overcome some limitations of the traditional modes of communication gives the impression of closeness in an isolated medium, similarly to informal language, which creates a sense of community among its users. This is common in certain Internet genres whereas in others there is a preservation of standard language features. Thus, the view of the Netspeak as a register has more weight than the one which sees it as a deviation of the standard language that must be fought back at any cost.

A register is a variety of language corresponding to a particular context whose three situational variables 'field', 'tenor', and 'mode' have significant and predictable impacts on language use (Lukin et al., 2008). The field refers to the topic dealt with (e.g., academic, technical, everyday), the tenor indicates the relationship between the interlocutors (e.g., power relationship, closeness, age, gender) and the mode (e.g., spoken, written, CMC), which change language to make it appropriate to each particular situation. Hence, due to the Netspeak mode's limitations and innovations, the audience to whom the message is addressed, the relationship between users and the topic discussed will adjust the linguistic and stylistic characteristics of the message. Since Netspeak is likely to have far-reaching consequences for a great majority of young people in the early twenty-first century, the importance of a new linguistic discipline such as Internet linguistics, "an approach to understanding how [online] language [works]" (Young, 2013), is vital to provide the future speakers and teacher of English with a theoretical framework of all these changes in vocabulary, grammar, and spelling in order to use the 'Netspeak register' appropriately.

\section{Methodology}

As argued by Randall (2002), deviations of Standard English are less noticeable in websites related to formal genres such as newspapers, criticism blogs or academic websites where the writer's message will resemble the standard features of written texts. According to this hypothesis, I collected a corpus of 527 posts from the microblogging platform Tumblr, regarded as a representative of an informal website accessed mainly by teenagers and young adults who interact freely, sharing and discussing information and personal experiences, and engaging in many-to-many and one-to-one conversations. The main purpose of this quantitative analysis was to explore the frequency with which linguistic deviations occur, in what kind of interactions appear and to reflect on whether they are as dangerous for the language as some scholars have argued. The discourse analysis focused on a detailed exploration of the standard and non-standard use of the grammatical features, discourse, style, typography and punctuation encountered in the corpus.

\section{Results}

The texts collected for the corpus, organised from higher to lower frequency, can be classified according to their format as text (45.92\%), image (23.72\%), comment (14.04\%), question (13.85\%), 
audio $(1.90 \%)$ and video $(0.57 \%)$. The comment and question formats are also popular and stress the interactivity of the website since users can add their opinions by editing the original post and ask/answer other users in one-to-one discussions. Following chat conversation and microblogging format conventions, messages tend to be short, users directly address in one-to-one or many-to-many conversations, and they discuss topics regarding generally filmic material (32.25\%), personal issues and opinions $(26.18 \%)$, and news $(9.11 \%)$ in a conversational way, which brings to the fore the emotive function of language (Peterson, 2011). Supporting previous studies on Internet language use (Crystal, 2001; Randall, 2002), several standard English features (51.70\%) can be seen in the use of grammar, typography and punctuation rules while non-standard English features represented $48.30 \%$ of the total analysed posts. Another popular element of the medium is the use of multimedia features (88.66\%) as an example of how the new features of CMC can supplement texts written in both standard and non-standard English by adding the tag system, images and gifs, and hyperlinks to provide a more precise contextualization.

\subsection{Discourse Analysis: grammar}

In agreement with Baron (2010), the most common grammatical features in the corpus texts were explicit references to the writer (50.09\%) and the reader (29.22\%) and the use of intensifiers. These three features reflect the importance of the users' interaction in the medium conveying a sense of inclusive community, and the relevance of the expressive function of language concerning emotions and interests. Unlike conventional writing, the writer/reader relationship in the corpus texts is a close one. The use of first person pronous reflects that the main topics in Tumblr are those related to personal issues. Thus, it is not surprising to find a high presence of the forms $I / \mathrm{my} / \mathrm{me}$ or we/our. The lattest is also frequently used as an inclusive pronoun, referring to a group of people interested in the same hobbies, and usually providing a sense of closeness and common goal e.g., we have literally created our own dialogue?, can we talk about.... Another form writers use to address their readers is by means of using expressions such as you, guys, people. You is used for three different functions: to address the reader, to generalise when a person says an example or a situation they imagine can be familiar to the reader, or to reinforce the vocative, e.g., all you guys know.

Repetition $(9.11 \%)$ and intensifiers $(29.03 \%)$ are also recurrent linguistic features in the corpus texts. Repetition mainly occurs in single words e.g., oh gosh oh gosh, really really, this... this photo man to convey emphasis and relevance. In the case of intensifiers the most frequent ones are so, really and very accompanying evaluative adjectives such as great, awesome, amazing, or situations where intensifiers can be replaced by slang vocabulary e.g., hella. Other intensifying forms are the structure how + adj; superlatives with the particle ever like the cuttiest thing I've ever seen; intensifying adverbs such as absolutely, totally, the over-use of intensifiers more super special awesome; and creative metaphors and exaggerations e.g., 10000\% better, the only news report anyone should care about or I wish it with the force of one thousand suns.

Another relevant grammar feature was the use of the indicative, interrogative and imperative modes. The imperative mode (11.39\%) is frequent in Tumblr posts, as in no listen this is actually, please write, send your Vikings confesion. At times it is informative, at other times it conveys the writer's excitement and subjectivity concerning the importance of the topic being discussedmeaning it should be compulsory. The interrogative mode is repeated several times throughout the corpus in expressions such as can we or can I simmulating the act of asking permission to talk about a topic, perhaps conveying the idea of immediacy and interactiveness, i.e. it is so important and obvious what the writer is going to express that it is unthinkable it has not been discussed yet. As a result, it is demonstrated that Tumblr may be a bit impositive community although at the same time politeness is present: please appears several times and compliments to the readers are present in many of the texts e.g., you guys are awesome. 
Moving to the structure of sentences, the average text is written with punctuation and paragraphs to give them a structure and cohesion, yet it is possible to see 'break sentences' and 'run-on sentences' in Tumblr. Break sentences (7.97\%) happen because of the influence of chats where almost every sentence is sent incomplete in order to achive quicker communication. An additional meaning for them is that they are another strategy for emphasis and for simplifying sentence structures. Run-on sentences $(3.80 \%)$ on the other hand, instantiate sentence constructions without any kind of punctuation. In the context of microblogging, they might be motivated because of the tag system where punctuation splits a tag or because their purpose is to immitate speech where pauses are made whenever needed, without the need of any punctuation sign. In the texts analysed they also suggest a sense of speed in conveying the message. In other words, they reflect ongoing discourse without any explicit pauses.

Regarding interjections (16.13\%), the most repeated words are confirmatory okay, meaning agreement and as a way to start the message. Onomatopoeias wow, ahh, pssst and conjuctions and or so are also typical features of spoken discourse (Biber et al., 2002), explicitly marking ongoing discourse. If writers address other users looking for information, answering previous posts, or they are aware of their audience, conventional greetings hello/hi, hey or vocatives dude, yo, it's okay are present at the beginning of the message.

Exemplification $(12.90 \%)$ is a strategy the writer uses to provide contextualization and to facilitate the reader the understanding of the message. They are introduced mainly by like and often combines with hedges, as in seems like or just like. Some expressions such as as, an example or including appear when dealing with academic and more formal topics. Another approach writers use to contextualize and add credibility to what they say is by quoting somebody's words (11.95\%). Direct speech — sometimes preceded by reporting verbs-is frequent within the medium when explicitly quoting somebody's words by means of inverted commas (11.95\%). The most recurrent reporting verbs are say, be like, tell, think, and go. The predominance of was like in the medium is because while said implies simply quotation, the former gives a general impression of the person being quoted, i.e. doing something very similar to the particular quoted material rather than focusing on phrasing details. In news or narrations where dialogue and action are present, other reporting verbs like "ask", reply, exclaim, shout are also used by writers to re-create the context.

\subsection{Discourse Analysis: discourse and style}

New lexicon on the Internet, leaving aside the vocabulary created because of the technological nature of the medium e.g., blogger, gif, or timeline, is mainly created by new expressions and words. The most common word formation methods found in the corpus are abbreviations and acronyms. Abbreviations (11.95\%) in the corpus can be:

1) sound-based $u$ 'you', cos 'because',

2) contractions wanna 'want to', kinda 'kind of',

3) missing termination of the original word congrats 'congratulations', anon 'anonymous' and

4) endings in -s or -ie pups 'puppies', hommie 'homeless'.

All of them are typical features of spoken language, so their use is not limited to CMC and its meaning is known for the majority of users. Analysing the acronyms (19.35\%) found in the corpus, they mainly coincide with what the literature describes as regards acronyms in Internet language (Crystal, 2001, pp. 85-86), and are representative of the different situations where they appear:

1) some acronyms are used in everyday conversation when referring to places USA or institutions $L G T B Q$,

2) accepted acronyms such as asap 'as soon as possible', or PS 'post-script',

3) a referential use to mention books, movies, series HP 'Harry Potter', GoT 'Game of Thrones' and 
4) acronyms of fixed expressions that help to save time when typing like omg 'oh my God', idk 'I don't know', lol 'laughing out loud', or tbh 'to be honest'.

Less common to find, compounds (2.28\%) are another word formation process related to terms created by the Internet birth. In Tumblr, words like askbox or fangirl can be easily seen as well as a new element called exclamation!compound e.g., young!Oberyn, which "suggests juxtaposition of two things; one of them is usually a person or character, to describe an alternate state of the subject" (allthingslinguistic.com, 2013). Blending (1.71\%) is commonly used to combine the names of two people although it is extended to other contexts too e.g., Brangelina 'Angelina Jolie and Brad Pitt', vlog 'video blog'. The clipping process (1.52\%) is also quite popular in the texts selected from the Tumblr website and words such as feels 'feelings', faves 'favourite', or defs 'definitively' are very frequent. Finally, the conversion method (3.61\%) often happens with nouns originated by new technologies, which now are used as verbs e.g., to blog, to email, to photoshop or to tweet.

In addition to word formation and the creation of new words, new ways of expression and communication (29.41\%) are popularised by youngsters in social networks mainly because of the use of puns, word plays, memes and specific types of writing (6.46\%) (Young, 2013). According to the Merrian-Webster Dictionary, an Internet meme is "an idea, behaviour, or style that spreads from person to person within a culture.” Examples of these meme expressions are also found in Tumblr in the form of fixed expressions such as mind blow, true story and friendzone; of particular images related to a specific idea that allow the modification of text on them; and the presence of certain writing styles like the 'Doge meme', described as an image accompanied by a deliberately form of broken English written in Comic Sans MS subunits.

Moreover, the analysis of the corpus shows that emotional language (21.63\%) recurred in the Tumblr posts. Onomatopoeias appear at the beginning of sentences functioning as interjections, or in the middle/end transmitting the writer's attitude wow and oh; conveying surprise; aaahh stands for understanding; awww means cute, adorable; haha is laugh; and yay means excitement. In addition to common specific constructions such as the use of

1) intensifiers want something so bad,

2) superlatives + ever be the best human be you can be,

3) exaggerations that child is the messiah or \#thanks to be one of those who sold their souls so you could exist and

4) evaluative remarks God is amazing;

There are verbs concerning positive and negative emotions e.g., love, makes me happy, upset, brought me to tears, references to religion $O H M Y G O D$, sweet Jesus; and the use of can has a remarkable role in Tumblr communication e.g. can't even explain, I have lost the ability to can. Actually, 'I Can't Even' is "an Internet slang expression used to indicate that the speaker is in a state of speechlessness, either as a result of feeling overjoyed or exasperated, depending on the context in which it is said." (Knowyourmeme.com, 2014). It is also important to mention that offensive language (14.61\%), as is also the case of informal and conversational language, is a recurrent feature in the corpus analysed. It mostly appeared in topics dealing with pastimes and opinions and the degree of offensiveness varies, from fuck, shit to freaking, bullshit or what the hell? They function as insults, modifiers fucking awesome and expressions of astonishment holy crap.

\subsection{Discourse Analysis: typography}

The most relevant aspects resulting from the corpus analysis regarding typography are the capitalisation use, italics, bold, and prolongation of vowels. By these means writers overcome some limitations of the written medium such as tone, rhythm, or any other phonological feature of communication. As many of the features previously discussed, these aspects can combine among themselves, be inconsistent and, especially in typography, intersperse between standard and non- 
standard English. For instance, the lack of capitalisation (32.83\%) often refers to those cases in which a text begins without a capital letter. No use of capitalization may mean that the writer does not follow the norm, even if it is the beginning of the sentence but later will do it after writing the next lines, or the writer uses capital letters after periods but she/he does not do so when referring to proper names.

Italics (9.30\%) and bold (8.16\%) typography are generally used in quotes and to highlight specific parts of a text. Bold is used to differentiate tittles from the body of the post or to emphasise certain words. Another way of putting emphasis on relevant fragments without using capital letters is when italics and bold are used at the same time. In the texts analysed, emphasis is also conveyed by adding letters to a word e.g., helloooo (5.31\%) and by using capitalisation on purpose (19.35\%) (Peterson, 2011). Within the Tumblr community context, when a post is written entirely with capital letters this is not considered as annoying as it would be in other genres. Here it indicates the writer is 'shouting' (emphasising) to share something they consider of vital importance-later it is up to the reader to consider whether it was so important or just nonsense. Capitalisation can appear as well in isolated words, expressions, or sentences the writer wants to emphasise. Depending on each situation, the purpose behind the capitalisation and prolongation of letters - the latter is frequently used in one-toone situations where one of the participants wants to give an impression of closeness, familiarity, etc.- varies, but it is mainly to attract the reader's attention to a specific part of the text.

Finally, in the sample of analysed texts it is possible to find two different types of misspellings, the ones that happen because writers do not proofread the text before sending it (1.70\%), as in civiilzation 'civilization', humaity 'humanity'; and when misspellings are done on purpose (10.44\%) because it is a way of showing that a user belongs to a certain group - with internal jokes and rules - and it imitates speech in a sort of simplified transcription: some are accepted wanna, yeah, y'all and others are not so frequent in the written medium imma follow dis, Da fuck they doin ova der, which become a main feature of meme writing.

\subsection{Discourse Analysis: punctuation}

Non-standard use of punctuation is also typical in the selected Tumblr texts. In agreement with Peterson's (2011) description of punctuation in the Internet register the aim of these features is to express emotions in a computer-mediated communicative situation, in which it is not possible to maintain face-to-face interaction. In other words, these features add tone, intensity, 'facial' expressions, contextualisation and a classification system. The most recurrent of all are use of multiple exclamations (12.33\%) and question marks, as in First impression: friends?!?!!? friends!!!!!!!! cute hair!!!!!!!!! or substituting the period (.).

Another key feature of CMC is missing punctuation (35.86\%) (Crystal, 2001). Often a text may initially follow the norm but later punctuation disappears. At other times, the standard use of the period (.) is substituted by exclamations because, as Crair (2013) notes, nowadays the full stop seems aggressive rather than neutral. Pauses may be done by breaking sentences or leaving them to the reader's decisions in run-on sentences. Similarly, the apostrophe may appear at the beginning of a text and later be forgotten \#made him into the hero he shouldnt be \#it's almost selfish of bucky.

The corpus findings also show that different types of spacing (3.80\%) convey different meanings such as urgency READ THIS. READ IT. LEARN IT. PREACH IT, intensification by separating letters of words just $s$ o goo $d$, or the opposite, the linking of words to express excitement and amusement AHHHKISSHIM!!, \#followme, a trend influenced by the tag system where in some websites such as Twitter, commas and periods split the tag into two. Finally, there is the possibility that non-standard spacing is done by a typing mistake when the writer does not check their text anotheractreesa role.

The second most recurrent punctuation symbol in the corpus is the hashtag (\#) (8.92\%) whereas the at (@) (1.52\%) appears mostly when naming a person in Twitter or Instagram “@KattWilliams”. As argued by Turner (2012), the hashtag has boosted its popularity in microblogs where trendy topics are created, and they help find topics on the Internet. In Tumblr, the tag system can appear outside or 
within the post and serves two main functions: to classify and organise posts in categories for an easier finding/avoiding of certain topics \#eurovision \#uk \#graham norton; and for writers to express their reactions towards a specific post \#i don’t even go hear \#but fdtd [from disk till dawn] has been showing up on my dash a lot now and then i get this and \#asjdhflgkjdslck [...].

The hashtag is very frequent in websites such as Instagram or Twitter where due to this strategy people try to get more popularity in the form of 'likes' and 'retweets'. Thus, as the Tumblr texts also illustrate, the use of tags as the main source of web organisation - in standard English posts too-is a characteristic of CMC that helps avoid the disappearance of posts with the passing of time.

Another feature found in the corpus is punctuation cross (1.33\%) e.g., I don't like shopping unless I'm buying myself my favourite food hehe and.... It shows the editing of the text with a comment the writer has eventually decided to delete although, contradictorily, it is still readable. It adds extra information that may be irrelevant but the writer wanted to share anyway. Parentheses often add extrainformation to the text e.g., an additional comment (but keep in mind I do have...) or the source of a post, whereas brackets (3.23\%) contextualise e.g., [Little girls runs and grabs his leg] Hello. Asterisks (3.42\%), in a similar way, are used for signalling non-verbal actions and contextualisation. It is another form used to insert comments that pull away the original text-backstage directions, contextualization, physical reaction (Yus, 2011) e.g., *cough*, *whispers*. Generally, there is only one word between the asterisks but nowadays descriptions become more detailed, which add richness and importance to the action e.g., *throws smoke bomb, disappears while youre distracted*, *imagines myself working out* okay that's enough exercise for the year.

Emoticons representing faces (7.59 \%) or objects (3.23\%) are also present in the corpus. As the literature explains, their purpose is 'to express the users' emotions producing positive judgments among users but sometimes they can alter the meaning of the message or even invalidating its propositional content altogether (irony, sarcasm...)” (Yus, 2011, p. 106). In Tumblr, emoticons are often used literally, matching with the writer's emotions. The most repeated smileys in Tumblr are happy faces :), sad faces :(, :O means surprise, and $\mathrm{xD}$ accompanies a humoristic comment. In the case of the non-facial emoticons, the most popular by far is $<3 \boldsymbol{\varphi}$, meaning love. Emoticons different from faces, usually have its own picture-in chat, instant messaging. The image can be added if the writer knows the web code to insert the emoticon; otherwise, it is easier to use the keyboard symbols.

\section{Conclusion}

The results of the present analysis are, broadly speaking, consistent with theoretical approaches that have described the main stylistic, linguistic and technological features of CMC and that have described Netspeak, a new mode of communication, as an amalgam of the written and spoken modes (Crystal, 2001; Peterson, 2011; Randall, 2002) with new approaches of expression and manipulation of language. The corpus has shown that the language used coincides with Crystal's description of "written speech" (2001) because it is mainly written although it reflects features of spoken language, particularly informal speech, which explains the linguistic deviations from standard English in the digital medium (Baron, 2010). Examples of distinctive features are the use of $I$ and you pronouns, interjections, intensifiers, emotional language and slang. Furthermore, the corpus analysis showed that the digital medium provides new features that ease the contextualization and clarification of the textual messages by means of multimedia elements or tags on the one hand, while on the other, it makes it possible to edit a text, and receive feedback easily.

Although the corpus has shown that some standard features of English are used in microblogging messages, to some extent contradicting the critics' complaints about the destruction of language (Crystal, 2001), linguistic innovations typical of Netspeak illustrate that, in fact, "the type of language that is being created online is affecting day-to-day speech patterns and writing styles of most young 
adults" (Baheri, 2013, p. 2). Some of the most recurrent features in the analysis correspond to the creation of new colloquialisms, acronyms, missing punctuation, non-standard use of capitalization, the use of new punctuation such as the hashtag, emoticons or asterisks, and the writing of phonological misspellings to imitate slang. Therefore, a field of research like 'internet linguistics' (Young, 2013) should keep on playing a role in the study of the Netspeak linguistic register and the issues of English as a Lingua franca in the digital medium.

Broadly, the selected corpus is illustrative of the way the Netspeak register works within a wide spectrum of formal and informal language that varies depending on the mode, tenor and field (Lukin et al., 2008) and that informal language often applies to genres of the Internet such as social networks, blogs, and microblogging platforms (Randall, 2002; Young, 2013; Yus, 2011) whose principal users are teenagers and young adults. The selected corpus, admittedly small, has shown that the texts in the microblogging context may be written in standard English, in standard English accompanied with multimedia files, a blending of standard and non-standard English features, or simply using nonstandard English features. Thus, the place where the communicative exchange takes place does not seem to determine the use of non-standard English, whose main aim is to foster speed in communication to shorten the time-lag (ElBekraoui, 2008).

The analysis of the corpus texts suggests that linguistic deviations tend to depend on the tenor and the relationship between the participants, who are the ones that decide the use of standard and nonstandard English features. The Tumblr texts have illustrated that questions and comments are the main post types in which non-standard English features appear. In questions, there is a direct one-to-one relationship between two people, usually sharing common interests and are familiar with their blogs, who engage into a question-answer pattern that many times includes greetings, emotive language, acronyms, exclamation marks and other features that resemble "written speech." On the other hand, the language used in comments and texts has proved to be more diverse since the audience the message is addressed is unknown and the topic discussed is serious or informal. In this particular case, the use of non-standard English may therefore switch from non-standard to standard, by this means avoiding the familiarity and closeness that non-standard English conveys. Field has also proved to be an important factor when defining the context of Netspeak communication. Tumblr users represent a community within which the most relevant topics dealt with are users' hobbies, personal experiences and opinions, all of them, also recurrent topics in informal speech. The data analysed has shown that in Tumblr, groups are created because of shared interests, which explains the recurrence of in-group references, shared humour and the use of linguistic deviations to overcome the constraints of tone and body language that the digital medium imposes. As Baheri (2013) puts it, users create their own 'Tumblr' argot, which enhances creativity and spreads the change and evolution of language.

It is hoped that the analysis of the present study has illustrated how the digital medium fosters the creation of a new language used in the Internet, sharing features with the spoken and written modes of communication and promoting its own stylistic devices. Language evolves and yields new genres, registers and conventions that require a renovation, or at least debate, of linguistic normative rules. The study shows that these linguistic novelties can be classified within the Netspeak register since they tend to be constrained by mode, field, and tenor. It is therefore important that research in the field of Internet linguistics further analyses language evolution and innovation as regards linguistic and stylistic features in order to determine whether the impact the Netspeak register may eventually lead, as some scholars have argued, to the destruction of language, or on the contrary, as an alternative path for linguistic evolution. 


\section{References}

Baheri, T. S. (2013). Your ability to can even: A defence of Internet linguistics. The Toast. Retrieved from http://the-toast.net/2013/11/20/yes-you-can-even/2/

Baron, N. (2010). Discourse structures in instant messaging: The case of utterance breaks. Language@Internet, 7.4, 1-26.

Biber, D., Conrad, S. \& Leech, G. (2002). The grammar of conversation. Longman student grammar of spoken and written English ( ${ }^{\text {th }}$ ed.). Harlow: Pearson Education Limited.

Crair, B. (2013). The period is pissed: When did our plainest punctuation mark become so aggressive? New Republic. Retrieved from http://www.newrepublic.com/article/115726/period-oursimplest-punctuation-mark-has-become-sign-anger

Crystal, D. (2001). Language and the Internet. Cambridge: Cambridge University Press.

ElBekraoui, M. L. (2008). The impact of the Internet on language. Theory of Electronic Design: Collected Papers. B. Hokanson (Ed.). Retrieved from https://wiki.umn.edu/pub/DHA5399/SyllabusSpring2010/DesignTheorySp2008.pdf

Exclamation!compounds. (2013). Allthingslinguistic.com. Retrieved from http://allthingslinguistic.com/post/46453848763/exclamation-compounds

I can't even. (2014). Knowyourmeme.com. Retrieved from http://knowyourmeme.com/memes/icant-even

Kleinman, Z. (2010). How the Internet is changing language. BBC News. Retrieved from http://archive.today/d2E3E

Lukin, A., Moore, A., Herke, M., Wegener, R., \& Wu, C. (2008). Halliday’s model of register revisited and explored. Linguistics and the Human Sciences. 4.2, 187-213.

Peterson, E. E. (2011). How conversational are weblogs? Language@Internet, 8.8, 1-18.

Randall, N. (2002). Lingo online: A report on the language of the keyboard generation. Retrieved from http://www.arts.uwaterloo.ca/ nrandall/LingoOnline-finalreport.pdf

Tagliamonte, S. A. (2008). Linguistic ruin? LOL! Instant messaging and teen language. American Speech 83.1, 3-34.

Turner, J. (2012). \#InPraiseOfTheHashtag. The New York Times. Retrieved from http://www.nytimes.com/2012/11/04/magazine/in-praise-of-the-hashtag.html?pagewanted=all\&_r=0

Young, N. (2013). Internet linguistics - Q\&A with David Crystal” Spark. Retrieved from http://sparkcbc.tumblr.com/post/52398439754/internet-linguistics-q-a-with-david-crystal

Yus, F. (2011). Cyberpragmatics: Internet-mediated Communication in Context. Google books. Amsterdam: John Benjamins. 